\title{
Effect of Oral Vitamin E on Oxidative Stress in Diabetic Kidney Disease囚A Protocol of Systematic Review and Meta-Analysis
}

\section{Renhong Wan ( $\sim 2499957477 @ q q . c o m$ )}

Tianjin University of Traditional Chinese Medicine https://orcid.org/0000-0002-1315-1997

\section{Pengwei Miao}

Tianjin Academy of Traditional Chinese Medicine Affiliated Hospital

\section{Yue Ji}

First Teaching Hospital of Tianjin University of Traditional Chinese Medicine

\section{Yihua Fan}

First Teaching Hospital of Tianjin University of Traditional Chinese Medicine

\section{Xiaohan Liu}

Tianjin University of Traditional Chinese Medicine

\section{Yuxin Fang}

University of Research on Experimental acupuncture science

\section{Xinju Li}

Tianjin University of Traditional Chinese Medicine

\section{Protocol}

Keywords: Diabetic kidney disease, Oral vitamin E, Oxidative stress, Systematic review protocol

Posted Date: March 1st, 2021

DOl: https://doi.org/10.21203/rs.3.rs-248713/v1

License: (c) (i) This work is licensed under a Creative Commons Attribution 4.0 International License. Read Full License 


\section{Abstract}

\section{Background}

The occurrence and progression of diabetic nephropathy is closely related to oxidative stress response. Vitamin E, as a lipid soluble antioxidant, has a long history of use. It may improve oxidative stress response to some extent, but there is no relevant support of evidence-based medicine. The purpose of this study was to systematically evaluate the effect of oral vitamin E on oxidative stress response in patients with diabetic nephropathy.

Methods

A computer was used for retrieving data from English database (PubMed, Embase, Web of Science, the Cochrane Library) and Chinese database (CNKI, Wangfang, VIP, CBMDISC) between the creation time to October 2020, a randomized controlled clinical study on oral vitamin E treatment of diabetic nephropathy. Two investigators conducted data extraction and literature quality evaluation for the included studies, and a Meta- analysis on the included articles was conducted using RevMan5.3 software.

\section{Conclusions}

This study will summarize up-to-date high-quality RCTs to assess the antioxidant effects of oral vitamin E on diabetic nephropathy. The findings of this study will help to determine whether oral vitamin $\mathrm{E}$ is effective and safe in patients with diabetic nephropathy.

Systematic review registration: PROSPERO CRD42020213945

\section{Introduction}

Diabetic kidney disease (DKD) is renal structural changes and functional abnormalities due to chronic microangiopathy in diabetes mellitus. About $30 \%-40 \%$ of diabetic patients will develop DKD [1], of which the main manifestations are early glomerular hyperfiltration, followed by the development of microalbuminuria to macroalbuminuria, decrease of glomerular filtration rate (GFR) [2], and end-stage renal disease, the end point of disease progression. Oxidative stress is an important risk factor for vascular complications in metabolic diseases, such as diabetes and obesity-related nephropathy[3]. After the body is stimulated by multiple pathological factors, excessive production of reactive oxygen species (ROS) in the body may compromise the oxidation resistance, and break the normal REDOX dynamic equilibrium, thus causing oxidative damage of biological macromolecules, such as proteins, lipids, nucleic acid, interfere with the normal life activities and form a state of severe stress. There is evidence indicating that the pathogenesis of diabetic nephropathy is closely related to oxidative stress[4], and the main mechanism for this is inflammation caused by hyperglycemia and oxidative stress response[5]. Experimental studies also clarified the role of oxidative stress in the occurrence and progression of DKD[6]. 
As a lipid soluble potent antioxidant[7], vitamin E can indirectly regulate and increase the activity of gSHPX in cells, thus inhibiting lipid peroxidation of the biofilm and maintaining the structural integrity of biofilm. Clinical studies have demonstrated that vitamin $\mathrm{E}$ coated dialyzers can improve chronic inflammation and oxidative stress without affecting dialysis adequacy[8], and animal studies have also confirmed the antioxidant and anti-apoptotic properties of vitamin $\mathrm{E}$, which may protect diabetic rats from myocardial and renal damage[9] and reduce the autophagy stress response[10].

Previous systematic review[11] summarized the effects of several antioxidants, including vitamin $E$, on diabetic nephropathy, but the included studies were limited, and there was great heterogeneity in the meta-analysis of different antioxidant studies. The systematic evaluation has not yet drawn conclusions with clinical guiding significance. Nevertheless, the antioxidant effects of vitamin $E$ in diabetic nephropathy have been shown to have great potential in this systematic evaluation. After this, a number of randomized controlled trials have confirmed that oral vitamin $\mathrm{E}$ can reduce oxidative stress in patients with diabetic nephropathy[12-14]囚but whether vitamin $E$ can be widely used in the treatment of diabetic nephropathy is still a question. Therefore, we intend to objectively and systematically evaluate the effect of oral vitamin E on oxidative stress response in diabetic nephropathy patients, so as to provide an evidence-based basis for clinicians.

\section{Methods}

\section{Study Registration}

This protocol of systematic review and meta-analysis has been drafted under the guidance of the preferred reporting items for systematic reviews and meta-analyses protocols (PRISMA-P). Moreover, the protocol and registration information are available at https://www.crd.york.ac.uk/prospero/\#searchadvanced (registration number: CRD42020213945).

\section{Inclusion criteria}

\section{Types of studies}

This study will include a randomized controlled trial of oral vitamin $E$ in the treatment of diabetic nephropathy. Language will be restricted to Chinese and English.

Types of participants

The diabetic nephropathy was definite (with diagnostic criteria based on the 2014 ADA Consensus)[15], without limitation on nationality, race, age, gender or course of disease.

\section{Types of interventions}

The test group received vitamin $\mathrm{E}$ or its derivatives orally, while the control group received other drugs orally or any form of placebo orally. 


\section{Types of outcome measures}

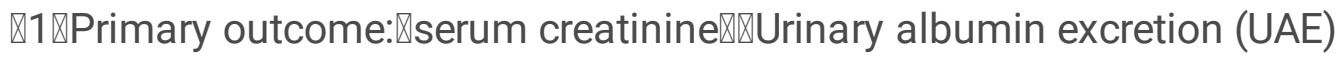

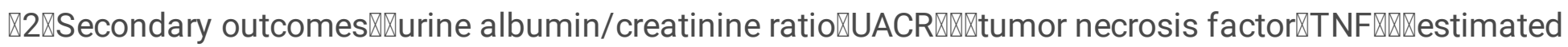

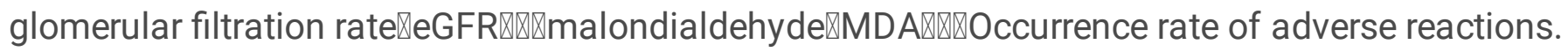

\section{Exclusion criteria}

(1) Repeated publications, for which only the most complete studies are include;

(2) Abstracts or articles with incomplete data, and articles of which complete data cannot be obtained after contacting the author;

(3) Articles evaluated as high risk of bias after randomization or hidden allocation[16] ;

(4) Test group or control group that are receiving dialysis treatment;

(5) Test group or control group that are receiving any other form of antioxidant (e.g. a-lipoic acid, vitamin C, etc.);

(6) Articles without related outcome indicators.

\section{Search methods for identification of studies}

"Vitamin E", "Antioxidants" $₫ "$ tocopherol" and "diabetic nephropathy" were used as Chinese terms for retrieval in Chinese databases, including CNKI, Wanfang Data Knowledge Service Platform, VIP, and CBMDISC. "Vitamin E”, "Tocopherol”,"Antioxidants"邓 “Diabetic nephropathy”, and "Diabetic Kidney disease" were used as English terms for retrieval in English database, including PubMed, EMBASE, Web of Science and the Cochrane Library. The retrieval time was from the creation time to October 2020, and all domestic and foreign articles on oral vitamin E treatment of diabetic nephropathy were collected. With PubMed as an example, and the retrieval strategy is as shown in Table S1.

\section{Data collection and analysis}

\section{Data extraction and management}

The data will be extracted independently by two investigators, the information will be recorded in the data extraction table, and their differences will be resolved with the help of a third reviewer. Detailed extraction information is as follows: $\triangle$ Clinical study (title, first author, publication date, sample size, sex ratio, average age, average course of disease); $\otimes$ Intervention measures for test group and control group (vitamin E dosage form, dose, frequency, course of treatment; type, frequency and course of placebo); $\mathbb{Z}$ Risk bias assessment factors in randomized controlled trials; $₫$ The outcome indicators required for the study. The literature screening process is as shown in Figure 1. 


\section{Assessment of risk of bias}

The Cochrane risk-of-bias tool will be used to assess the quality of included randomized controlled trials[17]. Two investigators will rate adequate sequence generatio, allocation concealment, binding, incomplete outcome date, selective reporting, other bias as low risk, unclear or high risk, as well as a cross check after completion of their respective assessment. If there are differences, there will be a discussion. If no agreement can be reached, there will be an investigator of the third party for consultation.

\section{Measures of treatment effect}

RevMan V.5.3 software will be used to synthesis all data. The results of dichotomous data will be presented as risk ratios and weighted mean differences with $95 \% \mathrm{Cls}$, while those of continuous data will be presented as standardised mean differences with $95 \%$ Cls.

\section{Dealing with missing data}

If the data for the required study is incomplete or not reported in the study, the investigators will contact the first author of the study or another author of the study by telephone or email. If the required data cannot be obtained, a descriptive analysis will be used instead of meta -analysis, and if necessary, these studies will be excluded.

\section{Unit of analysis issues}

The units of each outcome from different studies will be converted to the International System of Units before statistical analysis.

\section{Data synthesis}

RevMan5.3 software was used for Meta -analysis. Relative ratio (RR) was used for expression of dichotomous variables. For continuous outcomes, if the measurement tool was consistent with the measurement unit, the weighted mean difference (WMD) was used for the expression, otherwise the Standard Mean difference (SMD) was used as the effect quantity, and $95 \%$ confidence interval (Cl) was used for the expression. Heterogeneity was determined by $\chi^{2}$ and $P$ values, where ( $\left.P \geq 0.1, P \leq 50 \%\right)$ indicated low heterogeneity, for which a fixed-effect model was used for Meta- analysis, and $(P<0.1, R>$ $50 \%)$ indicated heterogeneity between studies, for which the source of heterogeneity should be analyzed. Clinical heterogeneity was treated by a subgroup analysis. If no significant clinical or methodological heterogeneity was found, statistical heterogeneity was considered, and the random-effect model was used for analysis. If the clinical heterogeneity was excessively obvious while the subgroup analysis could not be performed, only descriptive analysis was conducted, i.e. the meta- analysis was not performed.

\section{Subgroup analysis and investigation of heterogeneity}


If there is significant clinical heterogeneity between studies or $R^{2} \geq 50 \%$, a subgroup analysis will be required to explore the causes of heterogeneity. For example, subgroup analysis according to the concentration of oral vitamin E, subgroup analysis according to the stage of diabetic nephropathy, and subgroup analysis according to the course of treatment.

\section{Sensitivity analysis}

As recommended in the Cochrane handbook, a sensitivity analysis is needed for each indicator. In order to test the stability of meta-analysis results of indicators, a one-by-one elimination method will be adopted for sensitivity analysis.

\section{Assessment of publication bias}

Funnel plots were used to assess publication bias if no fewer than 10 studies were included in an outcome measure. Moreover, Egger's and Begg's test were used for the evaluation of potential publication bias.

\section{Evaluation of quality of evidence}

We will grade the outcome indicators through Grading of Recommendation Assessment, Development and Evaluation (GRADE)[18]. The evaluation includes bias risk, indirectness, inconsistency, inaccuracy and publication bias. The quality of evidence will be rated as high, medium, low or very low.

\section{Discussion}

The pathogenesis of diabetic nephropathy is complicated. It involves the disorder of glucose metabolism, abnormal hemodynamics and hemorheology. Gene, inflammation and oxidative stress are also important reasons for progression of DKD[19]. Presently, relevant studies have found that oxidative stress products are involved in various pathogenesis of DKD[4].The main mechanism of DKD kidney injury caused by

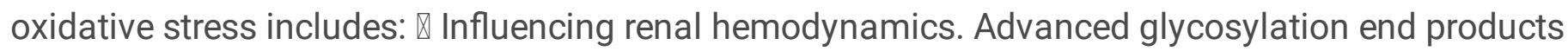
(AGEs), as combined with RAGE on mesangial cells, can induce production of reactive oxygen species (ROS) , thus mediating Ang $\otimes$ production increase. Under the condition of diabetes, ROS can be involved in occurrence of Intraglomerular hypertension through Ang $\otimes$. ROS can also affect the production of nitric oxide in the kidney, thus participating in Glomerular hyperperfusion and hyperfiltration[20]. $\otimes$ Being related to the damage of podocyte. High glucose leads to decreased synthesis of heparin sulfate in podocyte through ROS, thus resulting in loss of negative charge on basement membrane[21]; high glucose can also initiate podocyte apoptosis through ROS, induce the podocytes to fall off from the basement membrane, and reduce the number of podocytes in the glomeruli. खParticipating in the regulation of extracellular matrix (ECM). ROS, as a signal molecule, mediates the changes in renal cell behavior caused by high

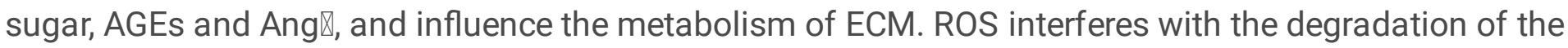
ECM through the t-PA/PAI system. खParticipating in the inflammatory response of the kidney. High sugar activates NF-KB through ROS, and NF-KB in turn upregulates McP-1 expression through promoting genetic 
transcription of monocyte chemotactic protein 1(McP-1)[22]. Therefore, the oxidative stress may be an important link or even the central link in the pathogenesis of DKD, and inhibiting oxidative stress may be a very important method for the prevention and treatment of DKD alone or in combination with other methods [23].

Vitamin E is a lipid soluble antioxidant that exists in cell membranes. Its main mechanism of action is affecting the lipid composition, stabilizing conjugated enzyme and preventing oxidative degradation of the membrane[24]. Animal studies have found that vitamin E can improve the imbalance between oxidative stress and antioxidant stress in diabetic rats, thus protecting the renal structure and function of diabetic rats[25]. The concomitant renal and systemic beneficial effects of vitamin E supplementation acting on multiple pathways, such as inflammation, vascular health, and oxidative stress have been confirmed clinically[26]. Vitamin E can inhibit the activation of NF-KB signaling pathway, regulate the production of $\mathrm{NO}_{2}-\mathrm{NO}_{3}$, increase the level of glutathione, reduce proteinuria and alleviate the damage of proximal renal tubules[27]. Some randomized controlled studies have shown that long-term high-dose vitamin E supplementation can significantly reduce the levels of TNF-a, MMP-2, MMP-9, malonaldehyde and end-terminal glycation products, and inhibit oxidative stress and inflammatory response; High dose vitamin E supplementation can reduce the levels of total cholesterol and low density lipoprotein cholesterol in blood, increase the total antioxidant capacity, and improve the renal function injury in DN patients[10 14].

Presently, it has been proved that the addition of vitamin E in a dialyzer may reduce oxidative stress and inflammation in dialysis patients with diabetic nephropathy[28] However, no consensus on the effect of oral vitamin $E$ on diabetic nephropathy has been reached. With the increase of relevant clinical trials, it is urgent to systematically evaluate the efficacy of oral vitamin $\mathrm{E}$ for improving oxidative stress in patients with diabetic nephropathy. In this study, we will summarize the latest evidence for oral vitamin $\mathrm{E}$ in the treatment of diabetic nephropathy. This is beneficial to both patients and decision makers in clinical practice.

It should be noted that the systematic evaluation may have some limitations. There may be some clinical heterogeneity due to differences in dose of oral vitamin and stage for the patients' conditions. At the same time, the duration of treatment may influence the outcomes. Due to the limitation in language ability, we have only searched literature in English and Chinese, and some other relevant studies or reports in other languages may be ignored.

Contributors This study was conceived by Pengwei Miao, Renhong Wan and Yihua Fan. Yihua Fan and Renhong Wan drafted the manuscript. Xiaohan Liu, Yue Ji囚and Yihua Fan participated in the design of the data synthesis analysis scheme. Pengwei Miao囚Renhong Wan reviewed and revised the manuscript.

\section{Declarations}

All authors have read and approved the publication of the protocol. 
Funding: This research was financially supported by the National Natural Science Foundation of China (No.81973944)

Competing interests: None.

Availability of data and materials

None

\section{Ethics approval and consent to participate}

Not applicable.

\section{Consent for publication}

Not applicable.

\section{Supplementary Material Information}

TableS1. Search strategy.

Funding/Support: This research was financially supported by the National Natural Science Foundation of China (No.81973944)

\section{References}

1 Umanath K, Lewis JB. Update on Diabetic Nephropathy: Core Curriculum 2018. American journal of kidney diseases : the official journal of the National Kidney Foundation 2018;71(6):884-95.

2 Alicic R, Rooney M, Tuttle KJCjotASoNC. Diabetic Kidney Disease: Challenges, Progress, and Possibilities. 2017;12(12):2032-45.

3 Sharma KJA, signaling r. Obesity and Diabetic Kidney Disease: Role of Oxidant Stress and Redox Balance. 2016;25(4):208-16.

4 Giacco F, Brownlee M. Oxidative stress and diabetic complications. Circulation research 2010;107(9):1058-70.

5 Liu Hongyan, Qiao Yufeng, Xue Fuping. New advances in targeted treatment of diabetic nephropathy by intervention oxidative stress pathway. J Chinese Journal of immunology. 2020;36(17):2174-78.

6 Sayed AA, Khalifa M, Abd el-Latif FF. Fenugreek attenuation of diabetic nephropathy in alloxan-diabetic rats: attenuation of diabetic nephropathy in rats. Journal of physiology and biochemistry 2012;68(2):2639. 
7 Abd Jalil A, Khaza'ai H, Nordin N, et al. Vitamin E-Mediated Modulation of Glutamate Receptor Expression in an Oxidative Stress Model of Neural Cells Derived from Embryonic Stem Cell Cultures. Evidence-based complementary alternative medicine 2017;2017:6048936.

8 Yang S, Xiao L, Xu B, et al. Effects of vitamin E-coated dialyzer on oxidative stress and inflammation status in hemodialysis patients: a systematic review and meta-analysis. 2014;36(5):722-31.

9 Abdel-Raheem MH, Salim SU, Mosad E, et al. Antiapoptotic and antioxidant effects of carvedilol and vitamin E protect against diabetic nephropathy and cardiomyopathy in diabetic Wistar albino rats. Hormone and metabolic research $=$ Hormon und Stoffwechselforschung $=$ Hormones et metabolisme 2015;47(2):97-106.

10 Zhao Y, Zhang W, Jia Q, et al. High Dose Vitamin E Attenuates Diabetic Nephropathy via Alleviation of Autophagic Stress. Frontiers in physiology 2018;9:1939.

11 Bolignano D, Cernaro V, Gembillo G, et al. Antioxidant agents for delaying diabetic kidney disease progression: A systematic review and meta-analysis. PloS one 2017;12(6):e0178699.

12 Aghadavod E, Soleimani A, Hamidi G, et al. Effects of High-dose Vitamin E Supplementation on Markers of Cardiometabolic Risk and Oxidative Stress in Patients with Diabetic Nephropathy: a Randomized Double-blinded Controlled Trial. Iranian journal of kidney diseases 2018;12(3):156-62.

13 Tan GCJ, Tan SMQ, Phang SCW, et al. Tocotrienol-rich vitamin E improves diabetic nephropathy and persists 6-9 months after washout: a phase lla randomized controlled trial. Therapeutic advances in endocrinology and metabolism 2019;10:2042018819895462.

14 Khatami PG, Soleimani A, Sharifi N, et al. The effects of high-dose vitamin E supplementation on biomarkers of kidney injury, inflammation, and oxidative stress in patients with diabetic nephropathy: A randomized, double-blind, placebo-controlled trial. Journal of clinical lipidology 2016;10(4):922-29.

15 Tuttle KR, Bakris GL, Bilous RW, et al. Diabetic kidney disease: a report from an ADA Consensus Conference. Diabetes care 2014;37(10):2864-83.

16 Zhao JG, Zeng XT, Wang J, et al. Association Between Calcium or Vitamin D Supplementation and Fracture Incidence in Community-Dwelling Older Adults: A Systematic Review and Meta-analysis. Jama 2017;318(24):2466-82.

17 Higgins JP, Altman DG, Gøtzsche PC, et al. The Cochrane Collaboration's tool for assessing risk of bias in randomised trials. BMJ (Clinical research ed) 2011;343:d5928.

18 Puhan MA, Schünemann HJ, Murad MH, et al. A GRADE Working Group approach for rating the quality of treatment effect estimates from network meta-analysis. BMJ (Clinical research ed) 2014;349:g5630. 
19 Sun YM, Su Y, Li J, et al. Recent advances in understanding the biochemical and molecular mechanism of diabetic nephropathy. Biochemical and biophysical research communications 2013;433(4):359-61.

20 Apakkan Aksun S, Ozmen B, Ozmen D, et al. Serum and urinary nitric oxide in Type 2 diabetes with or without microalbuminuria: relation to glomerular hyperfiltration. Journal of diabetes and its complications 2003;17(6):343-8.

21 Yard BA, Kahlert S, Engelleiter R, et al. Decreased glomerular expression of agrin in diabetic nephropathy and podocytes, cultured in high glucose medium. Experimental nephrology 2001;9(3):21422.

$22 \mathrm{Ha} \mathrm{H}, \mathrm{Yu}$ MR, Choi YJ, et al. Role of high glucose-induced nuclear factor-kappaB activation in monocyte chemoattractant protein-1 expression by mesangial cells. Journal of the American Society of Nephrology : JASN2002;13(4):894-902.

23 Li Qiang, An Ying, Sui Chunhong, et al. The role of antioxidants in the progression and treatment of diabetic nephropathy. J Chinese Journal of Integrated Traditional and Western Medicine nephropathy. 2016;17(03):275-76.

24 Fryer MJ. Vitamin E may slow kidney failure owing to oxidative stress. Redox report : communications in free radical research 1997;3(5-6):259-61.

25 Kedziora-Kornatowska K, Szram S, Kornatowski T, et al. Effect of vitamin E and vitamin C supplementation on antioxidative state and renal glomerular basement membrane thickness in diabetic kidney. Nephron Experimental nephrology 2003;95(4):e134-43.

26 Boaz M, Smetana S, Weinstein T, et al. Secondary prevention with antioxidants of cardiovascular disease in endstage renal disease (SPACE): randomised placebo-controlled trial. Lancet (London, England) 2000;356(9237):1213-8.

27 Yang H, Sun N, Li Y, et al. [Regulation of a-tocopherol on NFKB and Nrf2 signaling pathway at early stage of $\mathrm{N}$-nitrosomethylbenzylamine囚induced human esophageal cell carcinogenesis]. Zhonghua yu fang yi xue za zhi [Chinese journal of preventive medicine] 2015;49(6):546-53.

28 Yang SK, Xiao L, Xu B, et al. Effects of vitamin E-coated dialyzer on oxidative stress and inflammation status in hemodialysis patients: a systematic review and meta-analysis. Renal failure 2014;36(5):722-31.

\section{Figures}



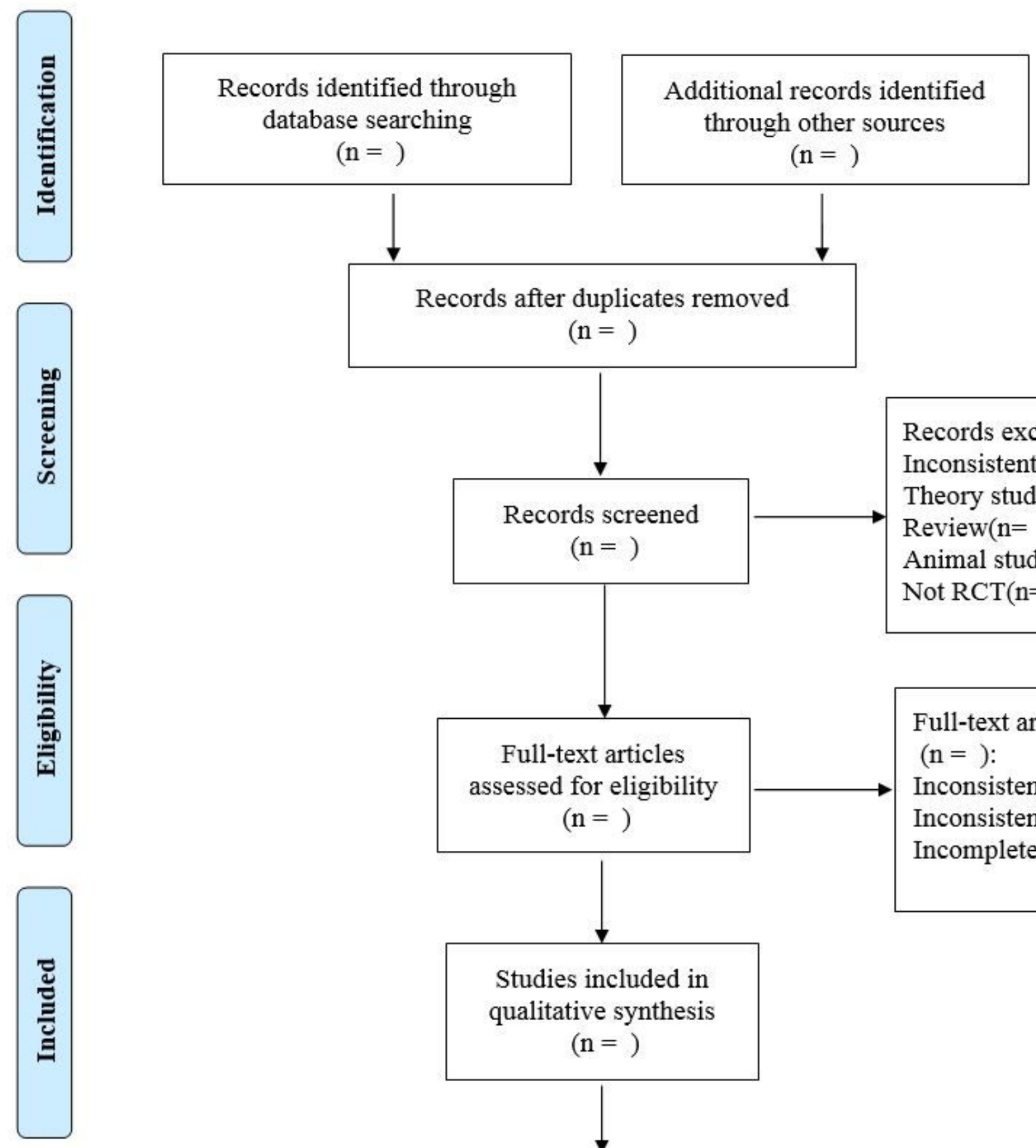

Records after duplicates removed $(n=)$
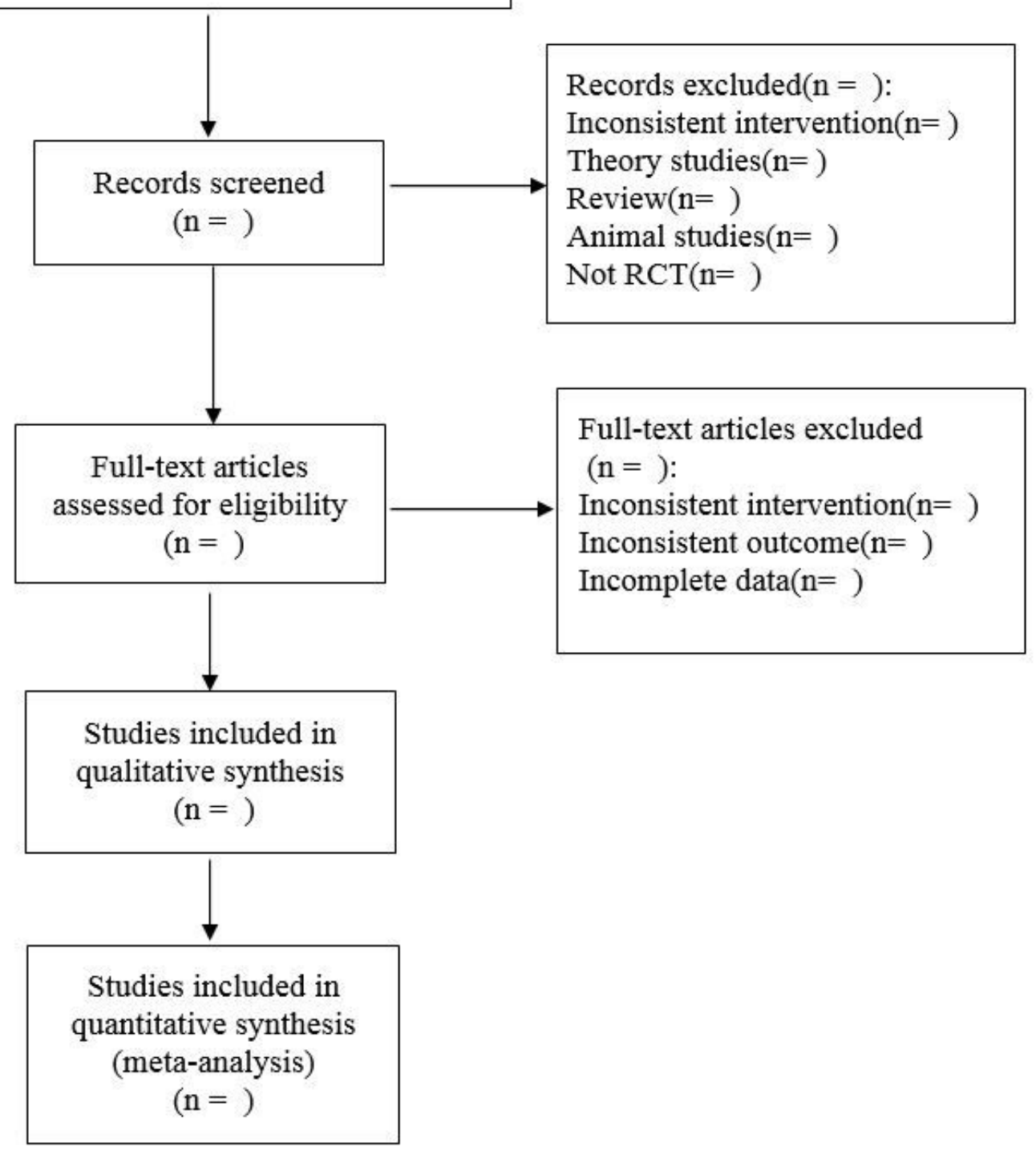

Figure 1

The literature screening process

\section{Supplementary Files}

This is a list of supplementary files associated with this preprint. Click to download. 
- PRISMAPchecklist.doc

- TableS1.Searchstrategy..docx 